\title{
Treatment with Methylphenidate for Attention Deficit Hyperactivity Disorder (ADHD) and the Risk of All-Cause Poisoning in Children and Adolescents: A Self-Controlled Case Series Study
}

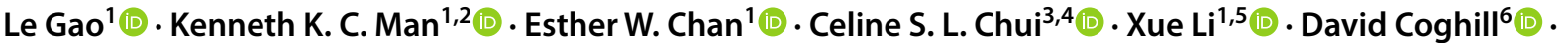

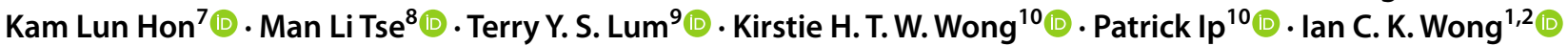

Accepted: 3 May 2021 / Published online: 20 July 2021

(c) The Author(s) 2021

\begin{abstract}
Background Children and adolescents with attention deficit hyperactivity disorder (ADHD) are at higher risk of all-cause poisoning by drugs and chemicals (intentional or accidental). Currently, there is limited data on whether medication treatment for ADHD can reduce the risk of all-cause poisoning.

Methods Patients aged 5-18 years with a methylphenidate (MPH) prescription and an incident poisoning diagnosis between January 2001 and June 2020 were identified from the Hong Kong Clinical Data Analysis and Reporting System. A selfcontrolled case series study design was used to compare the incidence rate ratios (IRRs) of all-cause poisoning during different risk windows (30 days before the first MPH prescription, exposure periods within 30 days of the first prescription, and periods of subsequent exposure) compared with the reference window (other non-exposure periods).

Results 42,203 patients were prescribed ADHD medication in Hong Kong during the study period. Of these, 417 patients who had both an MPH prescription and poisoning incident recorded were included in the main analysis. Compared with other non-exposed periods, a higher risk of poisoning was found in the 30 days before the first prescription (IRR 2.64, 95\% confidence interval [CI] 1.33-5.22) and exposure periods within 30 days of the first prescription (IRR 2.18, 95\% CI 1.06-4.48), but not during prolonged exposure. However, compared with 30 days before the first prescription as well as exposure periods within 30 days of the first prescription, there was a lower risk during the subsequent exposure (IRRs 0.49 and 0.60 , respectively). Similar results to the main analysis were also found in the subgroup analysis of intentional poisoning and females, but not in that of accidental poisoning and males.

Conclusions The risk of all-cause poisoning was higher shortly before and after the first MPH prescription and became lower during the subsequent prescription period. Our results do not support an association between the use of MPH and an increased risk of all-cause poisoning in children and adolescents and, in fact, suggest that longer-term use of MPH may be associated with a lower risk of all-cause poisoning, although this latter finding requires further study.
\end{abstract}

\section{Introduction}

Attention deficit hyperactivity disorder (ADHD) is a common neurodevelopmental disorder in children and adolescents [1,2]. It is estimated that around 5\% to $7 \%$ of children and adolescents worldwide [3] and 6.4\% in Hong Kong (HK) [4] are affected by ADHD. ADHD is frequently associated with various psychiatric comorbidities, including oppositional defiant disorder,

Le Gao and Kenneth K. C. Man have contributed equally as cofirst authors

Ian C. K. Wong

wongick@hku.hk

Extended author information available on the last page of the article anxiety, depression, substance misuse, and bipolar disorder [2, 5]. The core ADHD symptoms include inattention, hyperactivity and/or impulsivity $[2,6]$, and both core symptoms and comorbidities contribute to higher risks of injury [7-9], suicide $[10,11]$, and all-cause poisoning by drugs and chemicals (intentional or accidental) [12]. Some of these risks such as injury and accident have been proven to reach a particularly high level in youth (12-25 years) [13], which could be explained by the fact that the core symptoms may be more pronounced in children and adolescents [14], or that research on ADHD and accidents/ injuries have focused more on a specific age group (in young people) [13]. Poisonings due to medication and chemicals are very common in children and adolescents, with an estimated 45,000 deaths due to this cause worldwide every year [15]. Results from a cohort study showed that children and young 


\section{Key Points}

Current studies have explored the risk of all-cause poisoning in patients with attention deficit hyperactivity disorder (ADHD) versus those without ADHD, or reported poisoning cases from ADHD medication to poison centres. However, no population-based study has investigated whether medication treatment for ADHD can reduce the risk of all-cause poisoning.

This study showed that the risk of all-cause poisoning was higher shortly before and after the initiation of methylphenidate, followed by a decreased risk during the subsequent long-term methylphenidate treatment.

Along with previous ecological studies, safe storage and management of ADHD medication should still be emphasised to both patients and family members.

people with ADHD are at a four-fold higher risk of poisoning compared with those without ADHD [7]. Similar results were also reported in a recent meta-analysis, with a pooled relative risk of 3.14 from nine studies [12]. Notably, authors of the meta-analysis highlighted the need for further research focusing on the impact of medication treatment for ADHD on the risk of all-cause poisoning [12].

Stimulants such as methylphenidate (MPH) and amphetamines, and non-stimulants like atomoxetine, are common treatments for ADHD [16]. Over the past two decades, ADHD medication use has increased in many countries and regions, such as the United States (US), the United Kingdom, Australia and HK [17]. Ecological (analysis of secular trends) studies in the US [18] and Australia [19] observed an increasing trend of poisoning cases from ADHD medication reported to poison centres. However, it has remained unclear whether the increased use of pharmacological treatment of ADHD has a protective effect on the risk of overall poisoning.

Theoretically, ADHD medications could reduce the risk of poisoning in patients with ADHD by controlling core symptoms [20]. Hence, Ruiz-Goikoetxea et al. hypothesised that patients receiving ADHD medications are at a lower risk of all-cause poisoning [12]. Our study aims to test this hypothesis by comparing the risk of all-cause poisoning between MPH treatment and non-treatment periods.

\section{Methods and Materials}

\subsection{Database}

We obtained data through the Clinical Data Analysis and Reporting System (CDARS), an electronic database managed by the HK Hospital Authority (HA) that includes healthcare records since 1995 from all public hospitals and clinics in HK. It includes data from these health care facilities that are available to all $\mathrm{HK}$ residents ( $>7.3$ million) and covers approximately $80 \%$ of all hospital admissions in HK [21]; moreover, the majority of children who have chronic conditions are under the care of the HK HA [22]. The database includes inpatient, outpatient (ambulatory care), and accident and emergency (A\&E) department admission records. Information including diagnosis, hospital admissions and discharges, prescription and dispensing information is recorded and well protected by using deidentified patient ID. Data from CDARS has been validated and utilised for pharmacoepidemiological research [23-27]. The study protocol was approved by the Institutional Review Board of the University of Hong Kong/ Hospital Authority Hong Kong West Cluster (Reference No. UW 12-136).

\subsection{Study Design}

We used a self-controlled case series (SCCS) design to compare the incidence of the outcome of interest (poisoning), during the exposure (MPH treatment) period, with that in the non-exposure (non-treatment) period [28]. In SCCS studies, only patients who experienced both exposure and outcome are included. This within-individual comparison approach has several advantages as it controls for time-invariant confounders, both measured and unmeasured, that vary between individuals (i.e. genetic factors, disease severity, underlying comorbidities and socioeconomic status), and eliminates selection biases related to control groups that are common in cohort and case-control study designs that rely on betweenindividual comparison [29, 30].

\subsection{Patients Selection}

We selected children and adolescents aged 5-18 years who received at least one prescription of MPH and experienced incident poisoning during the period 1 January 2001 to 30 June 2020 from a cohort of individuals with ADHD medication prescriptions. Individual observation periods commenced at the study start date (1 January 2001), the first day of clinical record in CDARS, or 5th birthday, whichever was latest, and ended at the study end date (30 June 2020), one day before 19th birthday, prescription date of atomoxetine or the registered date of death, whichever was earliest. Patients with prescription(s) for atomoxetine were censored at their first atomoxetine prescription to avoid co-prescribing situations that may affect comparisons. MPH and atomoxetine were the only ADHD medications available in HK during the study period, and over $90 \%$ of patients were on MPH [31]. Therefore, this study has accounted for the majority of ADHD medication users in HK. We set the lower limit for 
follow-up at 5 years of age, as MPH is not recommended for younger children [16].

\subsection{Exposure}

The exposure window was defined as the periods of MPH use documented in the prescription records. If the prescription duration was not available, the prescribed quantity, frequency and strength were used to calculate the exposure window. Median imputation was applied for the missing duration of a very small proportion $(0.07 \%)$. We did not assume that patients received continuous treatment upon MPH initiation because clinicians may offer drug holidays to ADHD patients during school holidays [23, 25, 32]. Three cut-off points were used to define the individual study periods. Three risk windows (30 days before MPH was first prescribed, exposure periods within 30 days of the first prescription, and periods of subsequent exposure) and one reference window (other non-exposure periods) are defined in Fig. 1.

\subsection{Outcome}

The incident event was identified as the first poisoning event (all-cause, by drugs and chemicals, intentional or accidental) from the A\&E admission record, or the diagnosis records (including records from inpatient, outpatient, and $A \& E$ ) with the International Classification of Diseases, Ninth Revision, Clinical Modification (ICD-9-CM) code (E950-E952, intentional poisoning; E850-E869, 960-989, accidental poisoning). To ensure that the occurrences of poisoning events were independent of each other, only the first poisoning event was included in the main analysis. The date of the poisoning event was defined as the attendance/admission date of the hospital episode.

\subsection{Statistical Analysis}

Firstly, we did a descriptive analysis of the ADHD medication cohort from 2000 to 2020 by plotting the trend of the cumulative patient number with ADHD medication treatment as well as the number of poisoning episodes between 5 and 18 years old every 6 months. In SCCS analysis, incidence rate ratios (IRRs) and 95\% confidence intervals (CIs) were estimated by comparing the incidence rate of poisoning events during different risk windows and other non-exposure periods using conditional Poisson regression. We controlled for age at the 1-year age band and seasonal effect (cut-off points: 1 March, 1 May, 1 September, 1 November) in the analysis and used a 5\% significant level in all statistical analyses. The spline-based SCCS model was used to describe the exposure effect.
Using 0.25 as the ratio of the risk period to the whole study period, 339 poisoning cases will be required to detect an IRR of 1.4 with $80 \%$ power and 5\% significance level [33].

We also performed additional analyses to explore and validate our results. The diagnosis of otitis media was used as the negative control outcome for validation purposes. Currently, there is no evidence of a relationship between MPH and otitis media. If significant results are found, then unmeasured confounders might have been present and any significant findings in the main analysis would be questionable [34]. Subgroup analysis was conducted by sex and poisoning type (intentional poisoning and accidental poisoning). Sensitivity analyses included (i) extending the exposure periods by adding $1-10$ weeks after the end of each prescription period to assess the effect of non-adherence; (ii) adding a 7-day washout period after each exposure to ensure the effect of ADHD medication does not distort the baseline risk of poisoning (Online Resource Fig. S1, see electronic supplementary material [ESM]); (iii) including patients with both MPH prescription and ADHD diagnosis (the commencement of individual observation periods was revised at the start of the study period, first diagnosis date of ADHD, or on the patient's 5th birthday, whichever was latest); (iv) using the first prescription date of antidepressants or antipsychotics during the study period as the censor date to lessen the impact of these drugs on the poisoning outcome (detailed names of these drugs are shown in Online Resource Table S1, see ESM); (v) including all poisoning episodes of individuals who were in the main analysis and calculating the incidence of poisoning episodes during the study period-poisoning cases happening within 2 days were regarded as one poisoning episode; and (vi) re-setting the first medication use if a patient experienced a period without prescription longer than half a year or one year (Online Resource Fig. S2, see ESM).

Microsoft Excel $^{\circledR}, \mathrm{R}$ v3.6.1, and Statistical Analysis System $^{\circledR}$ (SAS) v9.4 (SAS Institute Inc., Cary, NC, USA) were used for data analysis. LG and KM conducted the analysis independently for quality control.

\section{Results}

We identified 42,203 individuals with ADHD medication prescriptions from CDARS, of which 38,968 had at least one prescription between the ages of 5 and 18 years old. During the past 20 years, the number of ADHD medication users aged 5-18 years and the number of poisoning episodes among this group of MPH users were increasing (Online Resource Fig. S3, see ESM). A total of 417 patients aged 5-18 years with an incident poisoning event and MPH prescription during the 
Fig. 1 Illustration of main selfcontrolled case series (SCCS) analysis. $M P H$ methylphenidate, $R x$ prescription
30 days before $1^{\text {st }} \mathrm{Rx}$

Other non-exposure period(s)

Exposure period(s) within 30 days of $1^{\text {st }} \mathrm{Rx}$

Subsequent exposure period(s)
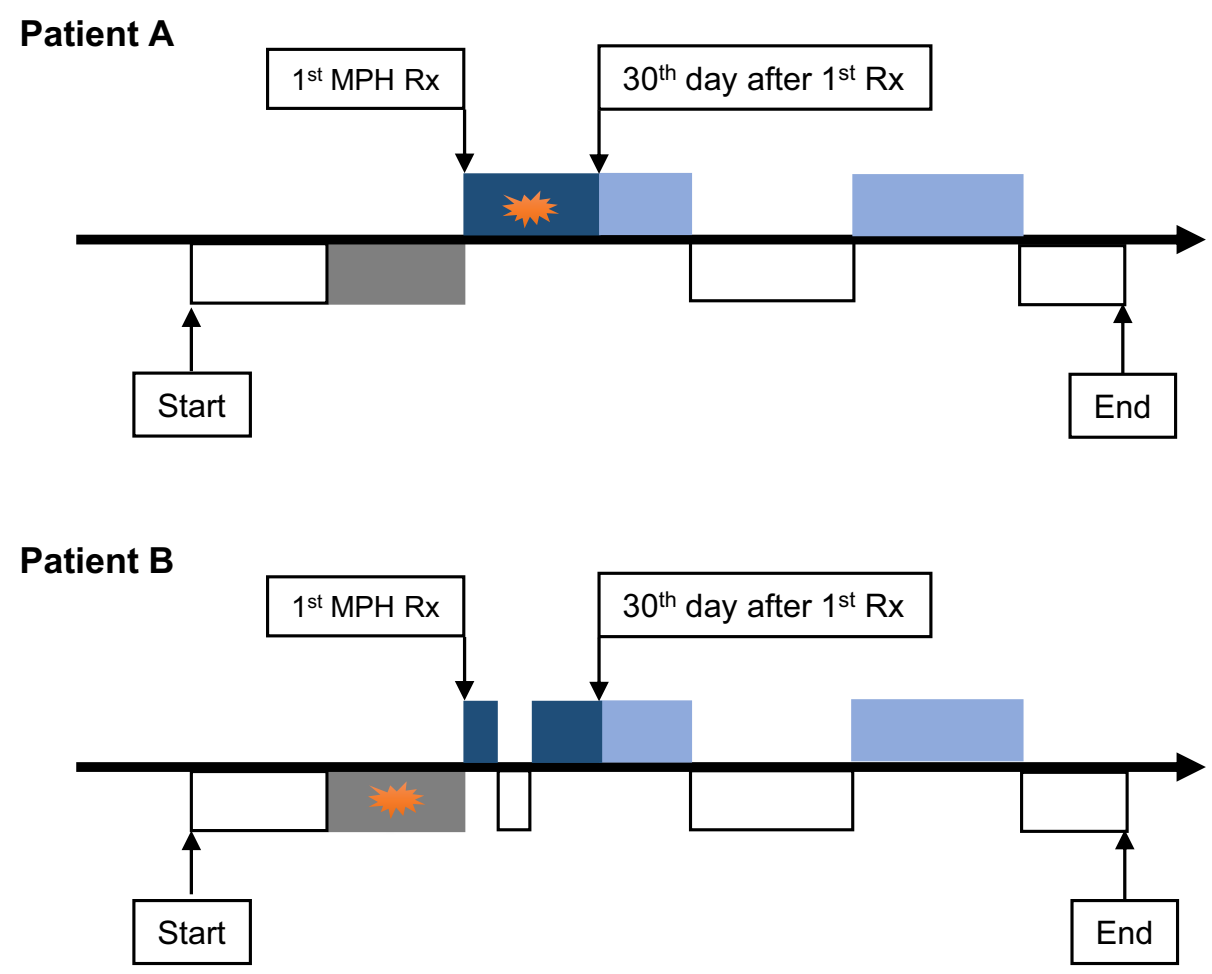

study period were included in the main analysis, and a total of 537 poisoning episodes identified in this group of individuals were included in the sensitivity analysis. The inclusion and exclusion steps of SCCS are shown in Fig. 2.

Baseline characteristics at the beginning of the observation period are listed in Table 1 . The mean age at the study start date was 6.06 years, while the mean age at first MPH use was 10.11 years and $106(25.4 \%)$ were female. The median duration of each prescription was 112 days, and the median duration of treatment was 1.89 years. There were no deaths recorded during the study period. Of the 417 patients, 318 (76.3\%) were diagnosed with ADHD, and $213(51.1 \%)$ had at least one record of other psychiatric disorders before or during the study period (Online Resource Table S2, see ESM). The overall incidence of poisoning episodes was 13.22 per 100 person-years during the study period (14.87 per 100 person-years in the MPH exposure period and 12.62 per 100 person-years in the non-exposure period).

\subsection{Main Self-Controlled Case Series (SCCS) Analysis}

Following adjustments for age and seasonal effect, a higher risk of all-cause poisoning was observed during the 30 days before the first MPH prescription compared with other nonexposure periods, with an IRR of 2.64 (95\% CI 1.33-5.22). Compared with other non-exposure periods, the risk of all-cause poisoning remained elevated during exposure periods within 30 days of the first prescription (IRR 2.18, 95\% CI 1.06-4.48) (Table 2). However, during the period of subsequent exposure, there was no statistically significant increase in risk compared with other non-exposure periods (IRR 1.30, 95\% CI 0.99-1.71). Comparison with the 30 days before the first prescription (Online Resource Table S3, see ESM) showed that there was no increase in risk during exposure periods within 30 days of the first prescription (IRR 0.83, 95\% CI 0.32-2.15). Furthermore, the risk of all-cause poisoning during the period of subsequent exposure was lower compared with that observed in the 30 days before the first prescription (IRR $0.49,95 \%$ CI 0.24-1.00), and was, although not statistically significant, lower than exposure periods within 30 days of the first prescription (IRR 0.60, 95\% CI 0.29-1.24). The splinebased figure showed that the risk of all-cause poisoning kept increasing before the first use of MPH and reached a peak at around 50 days before the first prescription (Online Resource Fig. S4, see ESM). 
Fig. 2 Patient inclusion flowchart. $A D H D$ attention deficit hyperactivity disorder, $M P H$ methylphenidate, SCCS selfcontrolled case series

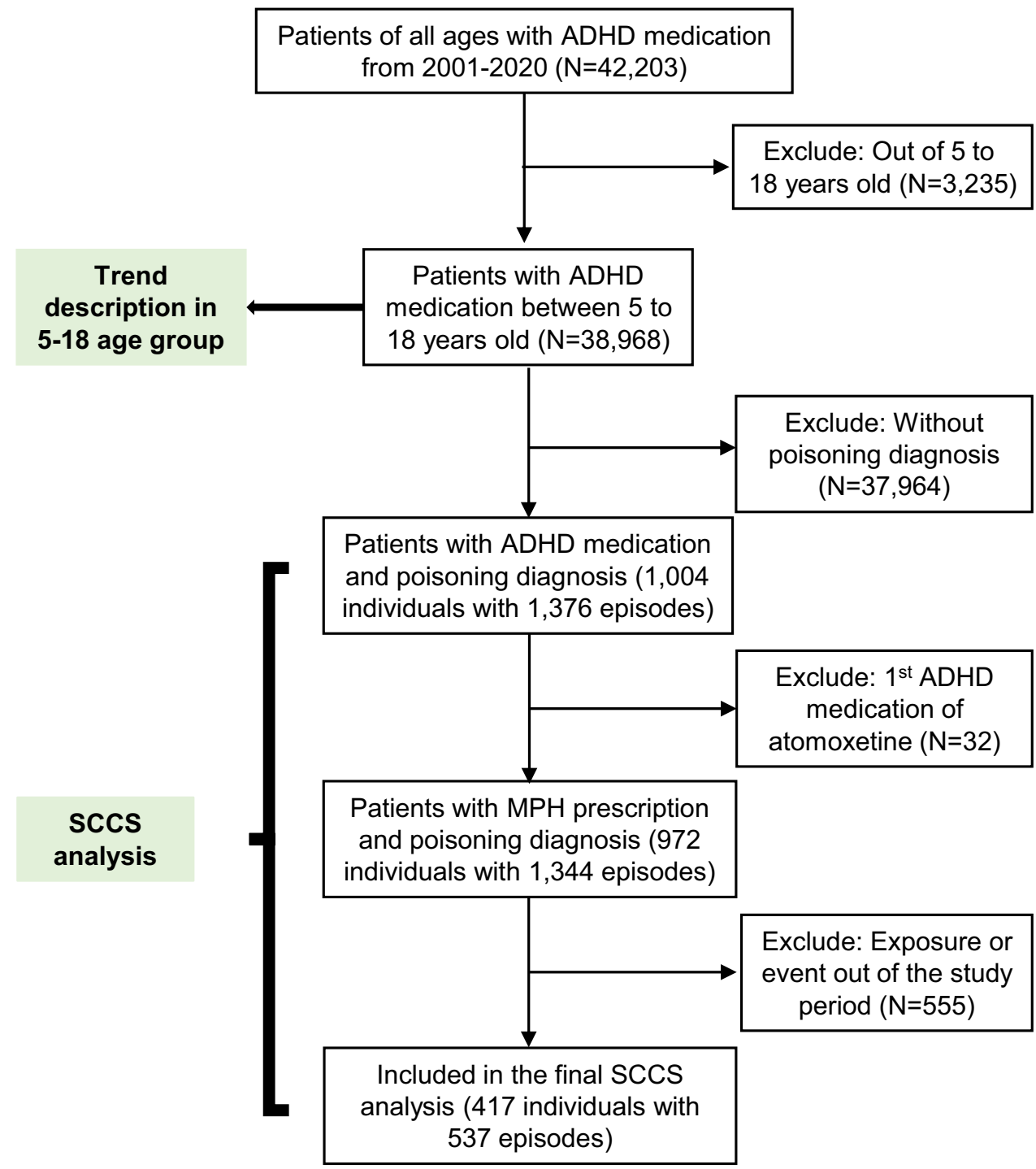

Table 1 Patients' characteristics in main SCCS analysis

\begin{tabular}{llll}
\hline Characteristics & Female & Male & Total \\
\hline No. of patients $(\%)$ & $106(25.42)$ & $311(74.58)$ & $417(100)$ \\
Mean age [y] at baseline $( \pm \mathrm{SD})$ & $6.45(2.70)$ & $5.95(1.84)$ & $6.06(2.06)$ \\
Mean age [y] of first prescription $( \pm \mathrm{SD})$ & $11.32(3.27)$ & $9.69(2.76)$ & $10.11(2.98)$ \\
Mean age [y] at poisoning event $( \pm \mathrm{SD})$ & $12.41(3.63)$ & $112(70-223.5)$ & $11.62(4.00)$ \\
Median days of each prescription (IQR) & $112(56-199)$ & $2.12(0.73-4.18)$ & $112(68-211)$ \\
Median years of MPH exposure (IQR) & $1.00(0.25-3.14)$ & & $1.89(0.48-3.89)$ \\
Exposed period & & 97 & 112 \\
$\quad$ No. of incident poisoning cases & 31 & 862.10 & 128 \\
No. of all poisoning episodes & 47 & & 1069.15 \\
Follow-up time (person-years) & 207.05 & 214 & 289 \\
Non-exposed period & 75 & 250 & 378 \\
No. of incident poisoning cases & 128 & 2209.43 & 2994.40 \\
No. of all poisoning episodes & 784.97 & & \\
Follow-up time (person-years) & & & \\
\hline
\end{tabular}

$I Q R$ interquartile range, $M P H$ methylphenidate, $S C C S$ self-controlled case series, $S D$ standard deviation 
Table 2 Results of main SCCS analysis of the incident poisoning episode $(N=417)$

\begin{tabular}{llll}
\hline Risk window & No. of events (follow-up person-years) & Adjusted IRRs (95\% CIs) & $p$-value \\
\hline Other non-exposure period(s) & $280(2962.86)$ & 1.00 & NA \\
30 days before first MPH Rx & $9(31.54)$ & $2.64(1.33-5.22)$ & 0.005 \\
Exposure period(s) within 30 days of first MPH Rx & $8(32.97)$ & $2.18(1.06-4.48)$ & 0.03 \\
Period(s) of subsequent exposure & $120(1036.18)$ & $1.30(0.99-1.71)$ & 0.06 \\
\hline
\end{tabular}

$C I s$ confidence intervals, IRRs incidence rate ratios, $M P H$ methylphenidate, $N A$ not applicable, $R x$ prescription, $S C C S$ self-controlled case series

\subsection{Additional Analyses}

The negative control analysis showed no association between MPH and otitis media, supporting the validity of our primary results (Online Resource Table S4, see ESM).

Subgroup analysis results are described in Table 3. For intentional poisoning, the risk was higher in 30 days before the first prescription and exposure periods within 30 days of the first prescription compared with the other non-exposure period, and decreased in the period of subsequent exposure compared with the period shortly before and after the first prescription (Online Resources Table S3, see ESM). For accidental poisoning, no significantly higher risk in the 30 days before the first prescription and exposure periods within 30 days of the first prescription compared with the other non-exposure periods was found. In the subgroup analysis by sex, the result for females was consistent with the main analysis. However, for males, there were no significant differences between any of the three risk windows when compared with other non-exposure periods with IRRs of 1.61, 1.47 , and 1.24, respectively. No interaction between sex and exposure was observed ( $p$-value for interaction $=0.17$ ).

The robustness of the main analysis was supported by the sensitivity analyses. IRRs were stable after adding 1-10 weeks to each exposure period and also the 7-day washout period (Online Resource Fig. S5 and Online Resource Table S5, see ESM). When we restricted the analysis to those with ADHD diagnosis, a higher risk was detected in exposure periods within 30 days of the first prescription; however, due to the limited sample size, no cases occurred in the 30 days before the first MPH prescription. Similar results were found when the prescription start date of antidepressant or antipsychotics was used as the censor date, including all poisoning episodes in the SCCS analysis as well as redefining a new start of MPH use (Online Resource Table S3 and Online Resource Table S5, see ESM).

\section{Discussion}

Despite a higher risk of all-cause poisoning in MPH users during 30 days before the first MPH prescription as well as exposure periods within 30 days of the first prescription, comparisons between other risk windows showed the risk was lowered in the period of subsequent exposure. These results do not support the notion that MPH use is associated with increased risk of intentional or accidental poisoning. The robustness of these results was supported by the sensitivity and negative control analyses.

Biologically, it is not possible for MPH to exert any effect prior to the commencement of the MPH treatment. One possible reason for the observed increase in risk during the period shortly before starting medication could be related to the core ADHD symptoms or the related comorbidities. The core symptoms and those psychiatric comorbidities can increase the risk of behavioural and cognitive problems, hence the poisoning. While the reasons for increased risk in exposure periods within 30 days of the first prescription may be similar to that of 30 days before the first prescription, it may also be the case that the higher risk during the initial prescribing period is related to the suboptimal treatment of ADHD symptoms during the titration of MPH. It is also possible that in some cases, a poisoning event triggered the referral for assessment and subsequent identification of underlying ADHD prompted the commencement of MPH. In contrast, with the selected negative control event in this study, we did not identify the same risk pattern. These findings lend support to the validity of the selection of our methods using SCCS analysis for this important public health issue.

We did not observe an immediate decrease in the risk of all-cause poisoning after MPH treatment initiation; however, the risk of all-cause poisoning in the subsequent prescription period was lower than in the two extra high-risk periods (i.e. 30 days before and after initiation of MPH). The findings are indeed consistent with Ruiz-Goikoetxea et al.'s hypothesis that MPH reduces the core ADHD symptoms, which may in turn reduce the risk of all-cause poisoning [12]. Similarly, the use of ADHD medication and its association with lower risk of unintentional [35] and physical injury [36] have also been suggested in previous studies. However, it is important to consider that the association is not necessarily causal. Further research is needed to validate our findings and evaluate the potential reasons for our observations.

Analysis of intentional poisoning showed a significantly higher risk in 30 days before the first prescription as well 
Table 3 Results of subgroup analysis

\begin{tabular}{|c|c|c|c|c|}
\hline Factor & Risk window & $\begin{array}{l}\text { No. of events } \\
\text { (follow-up person- } \\
\text { years) }\end{array}$ & Adjusted IRRs (95\% CIs) & $p$-value \\
\hline \multicolumn{5}{|l|}{ Analysis by poisoning type } \\
\hline \multirow[t]{4}{*}{ Intentional poisoning $(N=113)$} & Other non-exposure period(s) & $69(848.67)$ & 1.00 & NA \\
\hline & 30 days before first MPH Rx & $4(8.15)$ & $5.64(1.89-16.85)$ & 0.001 \\
\hline & Exposure period(s) within 30 days of first MPH Rx & $3(8.68)$ & $4.16(1.23-14.07)$ & 0.02 \\
\hline & Period(s) of subsequent exposure & $37(305.67)$ & $1.15(0.67-1.98)$ & 0.61 \\
\hline \multirow[t]{4}{*}{ Accidental poisoning $(N=273)$} & Other non-exposure period(s) & $188(1899.55)$ & 1.00 & NA \\
\hline & 30 days before first MPH Rx & $5(21.01)$ & $2.05(0.83-5.05)$ & 0.11 \\
\hline & Exposure period(s) within 30 days of first MPH Rx & $5(21.80)$ & $1.95(0.79-4.80)$ & 0.15 \\
\hline & Period(s) of subsequent exposure & $75(662.44)$ & $1.30(0.92-1.84)$ & 0.14 \\
\hline \multicolumn{5}{|l|}{ Analysis by sex } \\
\hline \multirow[t]{4}{*}{ Female $(N=106)$} & Other non-exposure period(s) & $70(776.89)$ & 1.00 & NA \\
\hline & 30 days before first MPH Rx & $5(8.08)$ & $5.58(2.13-14.62)$ & $<0.001$ \\
\hline & Exposure period(s) within 30 days of first MPH Rx & $4(8.11)$ & $4.38(1.54-12.46)$ & 0.006 \\
\hline & Period(s) of subsequent exposure & $27(198.94)$ & $1.61(0.90-2.88)$ & 0.11 \\
\hline \multirow[t]{4}{*}{ Male $(N=311)$} & Other non-exposure period(s) & $210(2185.97)$ & 1.00 & NA \\
\hline & 30 days before first MPH Rx & $4(23.46)$ & $1.61(0.59-4.37)$ & 0.35 \\
\hline & Exposure period(s) within 30 days of first MPH Rx & $4(24.86)$ & $1.47(0.54-4.00)$ & 0.45 \\
\hline & Period(s) of subsequent exposure & $93(837.24)$ & $1.24(0.90-1.70)$ & 0.19 \\
\hline
\end{tabular}

$C I s$ confidence intervals, IRRs incidence rate ratios, $M P H$ methylphenidate, $N A$ not applicable, $R x$ prescription

as exposure periods within 30 days of the first prescription compared with other non-exposure periods with wide CIs, which is due to the limited number of cases that occurred within these risk windows. It also showed a lower risk in the period of subsequent exposure compared with those two high-risk windows. The intentional poisoning cases were partly reported in our previous analysis on self-harm [25], which suggests a higher risk in the 90 days before and after the first MPH prescription. For accidental poisoning, the direction of the estimates is consistent with the main analysis. Again, such results do not support an association between accidental poisoning and MPH prescription.

In the subgroup analysis by sex, results in females were similar to the main analysis and, despite a larger sample size, no significant results were found in males. This suggests that the risk of poisoning is likely to be higher in girls. According to the public data, the reported incidence of poisoning (all causes) in females aged between 5 and 18 years is higher than for males in that age group $(0.07 \%$ vs $0.04 \%)[37,38]$. Additionally, sex differences in ADHD symptom severity may differ. For a variety of reasons, girls are less likely to be diagnosed with ADHD, and girls treated with MPH may have more severe symptoms [39, 40]. Unfortunately, we were not able to test this hypothesis due to the limitations of the dataset. However, according to the descriptive analysis in our study, more females (62.3\%) than males (47.3\%) had psychiatric comorbidities [41], which may explain the results.
Only $76 \%$ of the individuals of whom stimulants were prescribed had an ADHD diagnosis, which could be due to under-recording, or the need for MPH to control hyperactivity symptoms in subjects with autism spectrum disorder (ASD). However, our primary aim is to investigate if there is an association between the use of MPH and the risk of all-cause poisoning regardless of the indication of the medication. Also, we utilised a within-individual method so the baseline chronic conditions will not affect our study estimates. Therefore, our study conclusions were not affected by whether the included individuals had an ADHD diagnosis or not.

The main strength of our study is the use of the SCCS method, which relies on within-person comparison to balance fixed confounders as well as variable confounders of age and seasonal effect. Secondly, current research on the association between ADHD patients on medications and poisoning is limited, and so far, all studies are based on ecological data.

There are several limitations in this study. Firstly, we partly used the ICD-9-CM code to identify all-cause poisoning cases and classify the subtypes. Using ICD-9-CM may potentially affect the results by miscoding or misclassification, whereas there is no evidence that such miscoding will be affected by the MPH exposure. In such circumstances, it will reduce the sample size, hence the statistical power. Therefore, we identified the poisoning cases using 
two methods to ensure that as many poisoning cases as possible can be identified; one method was the ICD-9-CM code, the other was the A\&E admission record (extracted poisoning records of any poison type and poison nature). For the poisoning classification, although there are some diagnostic details in free text about the reason for poisoning, information was limited due to patient confidentiality; therefore, details of the specific chemicals or gas used for poisoning were not available. It is also likely that there were inconsistencies in the classification of whether a poisoning event was intentional or accidental. However, this study aims to examine the risk of poisoning regardless of the substance. Therefore, such limitations will not affect the conclusion of this study. Another limitation is the small sample size in some subgroups, so these analyses lacked statistical power. Further research using individual data from other populations is needed to confirm our findings and also evaluate the outcomes of other stimulant and also non-stimulant ADHD medications.

Our study does not support the hypothesis that the use of MPH in children and adolescents increases the risk of allcause poisoning; indeed, a lower risk was found in the period of subsequent treatment. This may provide some additional information for future guidelines, as prevention of poisoning has not been included in clinical practice guidelines as a benefit of ADHD pharmacotherapy. However, previous studies suggest that increased prescribing of ADHD medication is associated with an increased number of ADHD medication poisoning cases. Bringing all the current evidence together, a logical explanation to the increased poisoning cases with ADHD medication is that it could be related to poor storage and handling of such drugs. Epidemiological research has also shown that parents and siblings of children with ADHD are also more likely to have ADHD $[42,43]$ and this may increase the likelihood of poor storage and handling of MPH in the household. Therefore, in families with ADHD patients, family members with and without ADHD pharmacological treatment may face the risk of accidental poisoning due to ADHD medication. However, as there is currently a lack of detailed analysis of cases reported in different countries, we are unable to confirm our speculation. Therefore, further research on this topic is urgently needed to develop public health strategies to reverse the increasing occurrence of ADHD medication poisoning. In the meantime, we would like to emphasise educating carers and patients on the importance of safe handling and storage of MPH.

\section{Conclusion}

Children and adolescents prescribed MPH had a higher risk of all-cause poisoning shortly before and after the first prescription and the risk was reduced in the subsequent medication period. Our results do not support an association between the use of MPH and an increased risk of allcause poisoning in children and adolescents and, in fact, suggest that longer-term use of MPH may be associated with a lower risk of all-cause poisoning, although this latter finding requires further study.

Supplementary Information The online version contains supplementary material available at https://doi.org/10.1007/s40263-021-00824-x.

\section{Declarations}

Funding This work was supported by Hong Kong Research Grants Council General Research Fund (No. 17125419) to Kenneth K.C. Man, Esther W. Chan, Celine S.L. Chui, David Coghill, Kam Lun Hon, Man Li Tse, Patrick Ip, and Ian C.K. Wong. The University of Hong Kong provided funding for open access.

Conflict of interest Le Gao, Kam Lun Hon, Man Li Tse, Terry Y.S. Lum, and Kirstie H.T.W. Wong declare no conflict of interest; Kenneth K.C. Man is the recipient of the CW Maplethorpe Fellowship, and reports grants from the National Institute for Health Research, UK, the Research Grant Council, Hong Kong, and personal fees from IQVIA Ltd., outside the submitted work; Esther W. Chan has received honorarium from the Hospital Authority, research grants from the Narcotics Division of the Security Bureau of HKSAR, National Health and Medical Research Council (NHMRC, Australia), National Natural Science Foundation of China (NSFC), Research Fund Secretariat of the Food and Health Bureau (HMRF, HKSAR), Research Grants Council (RGC, HKSAR), Wellcome Trust, Amgen, AstraZeneca, Bayer, Bristol-Myers Squibb, Janssen, Pfizer, RGA and Takeda, outside the submitted work; Celine S.L. Chui reports grants from Pfizer and personal fees from Primevigilance, outside the submitted work; Xue Li reports grants from Health and Medical Research Fund (HMRF), Janssen and an internal Seed Fund from the University of Hong Kong, outside the submitted work; David Coghill reports grants and personal fees from Shire/Takeda, personal fees from Medice, personal fees from Servier, personal fees from Oxford University Press, outside the submitted work; Patrick Ip reports research grants from the Hong Kong Research Grant Council (RGC) and Health and Medical Research Fund (HMRF), outside the submitted work; Ian C.K. Wong reports research funding outside the submitted work from Amgen, BristolMyers Squibb, Pfizer, Janssen, Bayer, GSK, Novartis, the Hong Kong RGC and the Hong Kong Health and Medical Research Fund, National Institute for Health Research in England, European Commission, National Health and Medical Research Council in Australia, and also received speaker fees from Janssen and Medice in the previous 3 years.

Availability of data and material The datasets generated during and/or analysed during the current study are available from the corresponding author on reasonable request.

Code availability Same as data availability.

Ethics approval This study was approved by the Institutional Review Board of the University of Hong Kong/Hospital Authority Hong Kong West Cluster (Reference No. UW 12-136).

Consent to participate Not applicable.

Consent for publication Not applicable. 
Author contributions LG carried out the initial data analyses, drafted the initial manuscript, and had full access to the data in the study and takes responsibility for the integrity of the data and the accuracy of the data analysis; KKCM designed and conceptualized the study, crosschecked the analysis, reviewed the manuscript and had full access to the data in the study and takes responsibility for the integrity of the data and the accuracy of the data analysis; KHTW drafted the manuscript; EWC, CSLC, XL, DC, KLH, MLT and TYSL conceptualized the study, and critically reviewed the manuscript for important intellectual content; PI designed and conceptualized the study, coordinated and supervised data collection, and reviewed the manuscript; ICKW designed and conceptualized the study, coordinated and supervised data collection, reviewed the manuscript, and had full access to the data in the study and takes responsibility for the integrity of the data and the accuracy of the data analysis; All authors were involved in the acquisition, statistical analysis, or interpretation of data. All authors critically revised the manuscript for important intellectual content. All authors approved the final manuscript as submitted and agree to be accountable for all aspects of the work.

Open Access This article is licensed under a Creative Commons Attribution-NonCommercial 4.0 International License, which permits any non-commercial use, sharing, adaptation, distribution and reproduction in any medium or format, as long as you give appropriate credit to the original author(s) and the source, provide a link to the Creative Commons licence, and indicate if changes were made. The images or other third party material in this article are included in the article's Creative Commons licence, unless indicated otherwise in a credit line to the material. If material is not included in the article's Creative Commons licence and your intended use is not permitted by statutory regulation or exceeds the permitted use, you will need to obtain permission directly from the copyright holder. To view a copy of this licence, visit http://creativecommons.org/licenses/by-nc/4.0/.

\section{References}

1. Tso W, Chan M, Ho FK, Rao N, Li AM, Chan KL, et al. Early sleep deprivation and attention-deficit/hyperactivity disorder. Pediatr Res. 2019;85(4):449-55. https://doi.org/10.1038/ s41390-019-0280-4.

2. Leung AK, Hon KL. Attention-Deficit/Hyperactivity Disorder. Adv Pediatr. 2016;63(1):255-80. https://doi.org/10.1016/j.yapd. 2016.04.017.

3. Sayal K, Prasad V, Daley D, Ford T, Coghill D. ADHD in children and young people: prevalence, care pathways, and service provision. Lancet Psychiatry. 2018;5(2):175-86. https://doi.org/ 10.1016/S2215-0366(17)30167-0.

4. Liu A, Xu Y, Yan Q, Tong L. The prevalence of attention deficit/hyperactivity disorder among Chinese children and adolescents. Sci Rep. 2018;8(1):11169. https://doi.org/10.1038/ s41598-018-29488-2.

5. Pliszka SR. Psychiatric comorbidities in children with attention deficit hyperactivity disorder: implications for management. Paediatr Drugs. 2003;5(11):741-50. https://doi.org/10.2165/00148 581-200305110-00003.

6. Thapar A, Cooper M. Attention deficit hyperactivity disorder. Lancet. 2016;387(10024):1240-50. https://doi.org/10.1016/ S0140-6736(15)00238-X.

7. Prasad V, West J, Sayal K, Kendrick D. Injury among children and young people with and without attention-deficit hyperactivity disorder in the community: the risk of fractures, thermal injuries, and poisonings. Child Care Health Dev. 2018;44(6):871-8. https:// doi.org/10.1111/cch.12591.
8. Liou YJ, Wei HT, Chen MH, Hsu JW, Huang KL, Bai YM, et al. Risk of traumatic brain injury among children, adolescents, and young adults with attention-deficit hyperactivity disorder in Taiwan. J Adolesc Health. 2018;63(2):233-8. https://doi.org/10. 1016/j.jadohealth.2018.02.012.

9. Hurtig T, Ebeling H, Jokelainen J, Koivumaa-Honkanen H, Taanila A. The association between hospital-treated injuries and ADHD symptoms in childhood and adolescence: a follow-up study in the Northern Finland birth cohort 1986. J Atten Disord. 2016;20(1):3-10. https://doi.org/10.1177/1087054713486699.

10. Huang KL, Wei HT, Hsu JW, Bai YM, Su TP, Li CT, et al. Risk of suicide attempts in adolescents and young adults with attentiondeficit hyperactivity disorder: a nationwide longitudinal study. Br J Psychiatry. 2018;212(4):234-8. https://doi.org/10.1192/bjp. 2018.8.

11. Balazs J, Kereszteny A. Attention-deficit/hyperactivity disorder and suicide: a systematic review. World J Psychiatry. 2017;7(1):44-59. https://doi.org/10.5498/wjp.v7.i1.44.

12. Ruiz-Goikoetxea M, Cortese S, Magallon S, Aznarez-Sanado M, Alvarez Zallo N, Luis EO, et al. Risk of poisoning in children and adolescents with ADHD: a systematic review and meta-analysis. Sci Rep. 2018;8(1):7584. https://doi.org/10.1038/ s41598-018-25893-9.

13. Brunkhorst-Kanaan N, Libutzki B, Reif A, Larsson H, McNeill RV, Kittel-Schneider S. ADHD and accidents over the life span A systematic review. Neurosci Biobehav Rev. 2021;125:582-91. https://doi.org/10.1016/j.neubiorev.2021.02.002.

14. Balint S, Czobor P, Komlosi S, Meszaros A, Simon V, Bitter I. Attention deficit hyperactivity disorder (ADHD): genderand age-related differences in neurocognition. Psychol Med. 2009;39(8):1337-45. https://doi.org/10.1017/S00332917080042 36.

15. World report on child injury prevention. https://www.who.int/viole nce_injury_prevention/child/injury/world_report/en/ Accessed 20 May 2020.

16. NICE. Attention deficit hyperactivity disorder: diagnosis and management. National Institute for Health and Care Excellence: Clinical Guidelines. London: National Institute for Health and Care Excellence (UK); 2018.

17. Raman SR, Man KKC, Bahmanyar S, Berard A, Bilder S, Boukhris $\mathrm{T}$, et al. Trends in attention-deficit hyperactivity disorder medication use: a retrospective observational study using population-based databases. Lancet Psychiatry. 2018;5(10):824-35. https://doi.org/10.1016/S2215-0366(18)30293-1.

18. King SA, Casavant MJ, Spiller HA, Hodges NL, Chounthirath T, Smith GA. Pediatric ADHD medication exposures reported to US Poison Control Centers. Pediatrics. 2018;141(6). https://doi.org/ 10.1542/peds.2017-3872.

19. Cairns R, Daniels B, Wood DA, Brett J. ADHD medication overdose and misuse: the NSW Poisons Information Centre experience, 2004-2014. Med J Aust. 2016;204(4):154. https://doi.org/ 10.5694/mja15.00791.

20. Storebo OJ, Krogh HB, Ramstad E, Moreira-Maia CR, Holmskov M, Skoog M, et al. Methylphenidate for attention-deficit/hyperactivity disorder in children and adolescents: cochrane systematic review with meta-analyses and trial sequential analyses of randomised clinical trials. BMJ. 2015;351:h5203. https://doi.org/10. 1136/bmj.h5203.

21. Chan EW, Lau WC, Leung WK, Mok MT, He Y, Tong TS, et al. Prevention of dabigatran-related gastrointestinal bleeding with gastroprotective agents: a population-based study. Gastroenterology. 2015;149(3):586-95 e3. https://doi.org/10.1053/j.gastro. 2015.05.002.

22. Leung GM, Wong IO, Chan WS, Choi S, Lo SV, Health Care Financing Study G. The ecology of health care in Hong Kong. 
Soc Sci Med. 2005;61(3):577-90. https://doi.org/10.1016/j.socsc imed.2004.12.029.

23. Man KK, Chan EW, Coghill D, Douglas I, Ip P, Leung LP, et al. Methylphenidate and the risk of trauma. Pediatrics. 2015;135(1):40-8. https://doi.org/10.1542/peds.2014-1738.

24. Chui CS, Man KK, Cheng CL, Chan EW, Lau WC, Cheng VC, et al. An investigation of the potential association between retinal detachment and oral fluoroquinolones: a self-controlled case series study. J Antimicrob Chemother. 2014;69(9):2563-7. https:// doi.org/10.1093/jac/dku145.

25. Man KK, Coghill D, Chan EW, Lau WCY, Hollis C, Liddle E, et al. Association of risk of suicide attempts with methylphenidate treatment. JAMA Psychiat. 2017;74(10):1048-55. https://doi.org/ 10.1001/jamapsychiatry.2017.2183.

26. Lau WC, Chan EW, Cheung CL, Sing CW, Man KK, Lip GY, et al. Association between dabigatran vs warfarin and risk of osteoporotic fractures among patients with nonvalvular atrial fibrillation. JAMA. 2017;317(11):1151-8. https://doi.org/10.1001/ jama.2017.1363.

27. Man KKC, Lau WCY, Coghill D, Besag FMC, Cross JH, Ip P, et al. Association between methylphenidate treatment and risk of seizure: a population-based, self-controlled case-series study. Lancet Child Adolesc Health. 2020;4(6):435-43. https://doi.org/ 10.1016/S2352-4642(20)30100-0.

28. Petersen I, Douglas I, Whitaker H. Self controlled case series methods: an alternative to standard epidemiological study designs. BMJ. 2016;354:i4515. https://doi.org/10.1136/bmj.i4515.

29. Whitaker HJ, Farrington CP, Spiessens B, Musonda P. Tutorial in biostatistics: the self-controlled case series method. Stat Med. 2006;25(10):1768-97. https://doi.org/10.1002/sim.2302.

30. Nordmann S, Biard L, Ravaud P, Esposito-Farese M, Tubach F. Case-only designs in pharmacoepidemiology: a systematic review. PLoS ONE. 2012;7(11):e49444. https://doi.org/10.1371/journal. pone.0049444.

31. Man KK, Ip P, Hsia Y, Chan EW, Chui CSL, Lam MPS, et al. ADHD drug prescribing trend is increasing among children and adolescents in Hong Kong. J Atten Disord. 2017;21(14):1161-8. https://doi.org/10.1177/1087054714536047.

32. Man KK, Coghill D, Chan EW, Lau WC, Hollis C, Liddle E, et al. Methylphenidate and the risk of psychotic disorders and hallucinations in children and adolescents in a large health system. Transl Psychiatry. 2016;6(11):e956. https://doi.org/10.1038/tp.2016.216.

33. Musonda P, Farrington CP, Whitaker HJ. Sample sizes for selfcontrolled case series studies. Stat Med. 2006;25(15):2618-31. https://doi.org/10.1002/sim.2477.
34. Lipsitch M, Tchetgen Tchetgen E, Cohen T. Negative controls: a tool for detecting confounding and bias in observational studies. Epidemiology. 2010;21(3):383-8. https://doi.org/10.1097/EDE. 0b013e3181d61eeb.

35. Ruiz-Goikoetxea M, Cortese S, Aznarez-Sanado M, Magallon S, Alvarez Zallo N, Luis EO, et al. Risk of unintentional injuries in children and adolescents with ADHD and the impact of ADHD medications: a systematic review and meta-analysis. Neurosci Biobehav Rev. 2018;84:63-71. https://doi.org/10.1016/j.neubiorev.2017.11.007.

36. Man KKC, Ip P, Chan EW, Law SL, Leung MTY, Ma EXY, et al. Effectiveness of pharmacological treatment for attention-deficit/hyperactivity disorder on physical injuries: a systematic review and meta-analysis of observational studies. CNS Drugs. 2017;31(12):1043-55. https://doi.org/10.1007/ s40263-017-0485-1.

37. Population By-census. https://www.bycensus2016.gov.hk/en/bcmt.html Accessed 2 April 2020.

38. Lau KK, Chow TYA, Chan CK, Chan YC, Ng CHV, Ng SH, et al. Hong Kong Poison Information Centre: annual report 2017. Hong Kong J Emerg Med. 2018;25(6):313-23.

39. Quinn PO, Madhoo M. A review of attention-deficit/hyperactivity disorder in women and girls: uncovering this hidden diagnosis. Prim Care Companion CNS Disord. 2014;16(3). https://doi.org/ 10.4088/PCC.13r01596.

40. Ramtekkar UP, Reiersen AM, Todorov AA, Todd RD. Sex and age differences in attention-deficit/hyperactivity disorder symptoms and diagnoses: implications for DSM-V and ICD-11. J Am Acad Child Adolesc Psychiatry. 2010;49(3):217-28 e1-3.

41. Hinshaw SP. Academic underachievement, attention deficits, and aggression: comorbidity and implications for intervention. J Consult Clin Psychol. 1992;60(6):893-903. https://doi.org/10.1037// 0022-006x.60.6.893.

42. Starck M, Grunwald J, Schlarb AA. Occurrence of ADHD in parents of ADHD children in a clinical sample. Neuropsychiatr Dis Treat. 2016;12:581-8. https://doi.org/10.2147/NDT.S100238.

43. van Steijn DJ, Richards JS, Oerlemans AM, de Ruiter SW, van Aken MA, Franke B, et al. The co-occurrence of autism spectrum disorder and attention-deficit/hyperactivity disorder symptoms in parents of children with ASD or ASD with ADHD. J Child Psychol Psychiatry. 2012;53(9):954-63. https://doi.org/10.1111/j. 1469-7610.2012.02556.x.

\section{Authors and Affiliations}

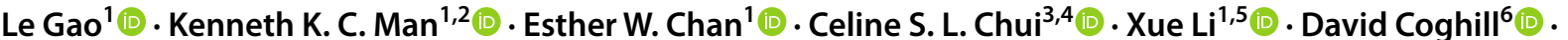

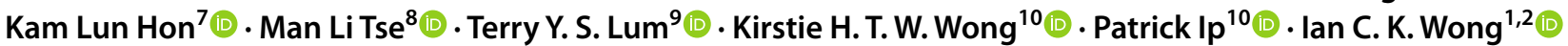

1 Department of Pharmacology and Pharmacy, Centre for Safe Medication Practice and Research, Li Ka Shing Faculty of Medicine, The University of Hong Kong, 2/F Laboratory Block, 21 Sassoon Road, Hong Kong, China

2 Research Department of Policy and Practice, Centre for Medicines Optimisation Research and Education, University College London School of Pharmacy, London, UK
3 School of Nursing, Li Ka Shing Faculty of Medicine, The University of Hong Kong, Hong Kong, China

4 School of Public Health, Li Ka Shing Faculty of Medicine, The University of Hong Kong, Hong Kong, China

5 Department of Medicine, Li Ka Shing Faculty of Medicine, The University of Hong Kong, Hong Kong, China 
6 Departments of Paediatrics and Psychiatry, Faculty of Medicine, Dentistry and Health Sciences, University of Melbourne, Melbourne, VIC, Australia

7 Department of Paediatrics, Faculty of Medicine, The Chinese University of Hong Kong, Hong Kong, China

8 Hong Kong Poison Information Centre, United Christian Hospital, Hong Kong, China
9

Department of Social Work and Social Administration, The University of Hong Kong, Hong Kong, China

10 Department of Paediatrics and Adolescent Medicine, Li Ka Shing Faculty of Medicine, The University of Hong Kong, Hong Kong, China 